\title{
Computational Study on Turbulence for a Flow through a Ring
}

\author{
Bhargav. $\mathrm{A}^{\dot{\mathrm{A}}^{*}}$, Krupakar Pasala ${ }^{\dot{\mathrm{A}}}$, Anil Kumar. $\mathrm{V}^{\dot{\mathrm{A}}}$ and Srinivasa Rao.P $\mathrm{P}^{\dot{\mathrm{A}}}$ \\ ${ }^{\dot{A}}$ Dept. of Aeronautical Engineering, Vardhaman College of Engineering, Hyderabad and GEWS Technical Foundations,
}

Accepted 10 January 2014, Available online 01 February 2014, Special Issue-2, (February 2014)

\begin{abstract}
Simulation of turbulence flow over a ring is connected using $k$-epsilon and k-omega methods, In order to investigate the flow characteristics over the ring. In case of sphere at $R_{e}=3000$, the shear layer is elongated in the direction of flow and forms a cylindrical vortex sheet and its instability begins to appear at $x \approx 2 d$. But in case of ring at $R_{e}=10^{5}$, the instability of shear layer occurs right behind the ring and near wake, the flow becomes turbulent.
\end{abstract}

Keywords: Turbulence, Reynolds number, Wakes, Vortex sheet

\section{Introduction}

Unsteady behavior can be observed behind any object even at low Reynolds numbers (Sakamoto and Haniu 1990; Johnson and Patel 1999; Mittal 1999). Therefore flow through a ring has not been well understood when compared to that over a sphere, even though the ring geometry is simple. Previous studies on flow over ring have been mostly based on experimental methods, using flow visualization vertical structures behind the ring is presented and the low frequency and high frequency characteristics in shear layer. When Re-exceeds about 1000 , the wake flow behind the ring changes from laminar to turbulent flow. A few numerical studies have been made on turbulent flow over ring, however detailed information on statistics of turbulence behind the ring is still limited in the literature.

The objective of the present paper is to investigate the turbulent flow characteristics over ring at different Reynolds numbers $3000 \& 10^{5}$ based on the velocity of free-stream $u_{\infty}$ and the external diameter of ring $d$.

\section{Numerical method and Computational Setup}

The numerical method used is based on immersed boundary method in a cylindrical coordinate. Here ring in the flow is treated as forcing momentum in the N-S equations. Therefore, flow over the ring can be easily handled with cylindrical grids (orthogonal) that do not coincide with the surface body. At two different Reynolds numbers numerical simulations are performed. The Reynolds numbers are $\mathrm{R}_{\mathrm{e}}=\mathrm{u}_{\infty} \mathrm{d} / \mathrm{v}=3000$ and $10^{5}$. The computational domain used is $-15 \mathrm{~d} \leq \mathrm{x} \leq 15 \mathrm{~d}, 0 \leq \mathrm{r} \leq 15 \mathrm{~d}$ and $0 \leq \theta \leq 2 \pi$. The number of grid points is $75(\mathrm{x}) \mathrm{x} 81(\mathrm{r}) \mathrm{x} 40(\theta)$. Here $v, r$ and $x$ respectively denote the azimuthal, radial

*Corresponding author: Bhargav.A

DOI: http://dx.doi.org/10.14741/ijcet/spl.2.2014.90 and stream-wise directions. Finally 461701 quadrilateral wall faces, 911416 triangular interior faces, 876520 nodes are generated. The grid which displays the ring and the surface is attached.

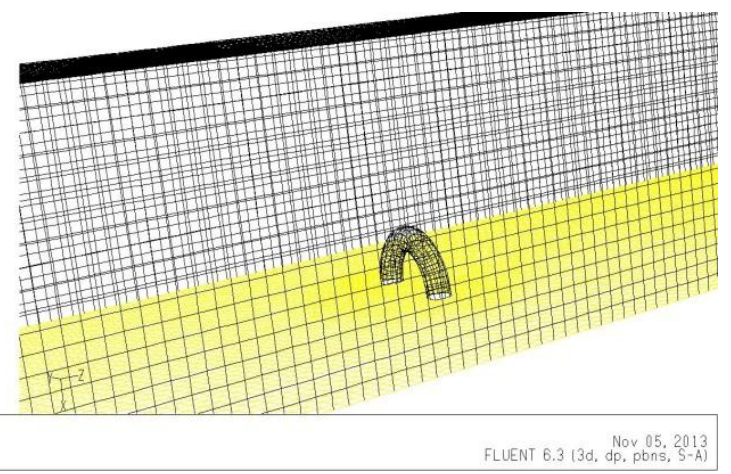

Fig.1 Grid of the ring and the surface

At high Reynolds number, for convection dominated flow serious dispersion errors were created by second order central difference scheme and hence in the region of velocity acceleration 2- $\Delta$ waves appear (at leading surface of ring for example). Two different approaches have been taken to resolve this problem. One approach is to apply a filtering procedure which helps in adding numerical dissipation in the manner of an adhoc and the other approach is to use upwind-biased schemes. However in these approaches, the numerical dissipation will become larger than the subgrid-scale velocity dissipation (Beaudan and $\mathrm{MO}_{\text {in }}$ 1994).

A hybrid approach for flow over a bluff body had proposed for the spatial discretization of the convection terms. In regions of laminar accelerating flow, a high order up-wind scheme is used and the central difference scheme of second order is used elsewhere. With the help of second-order central difference scheme, the diffusion 
terms are discretized. With this approach, there is a possibility of avoiding contamination of solutions resulting from the dissipative schemes application to the whole flow filed.

\section{Mathematical Modeling}

The dimensionless form of Navier-Stokes equations for Newtonian Viscous fluid is

$$
\frac{\partial u}{\partial t}+u \cdot \nabla u+\nabla p-\frac{1}{R e} \nabla^{2} u=0, \nabla \cdot u=0
$$

$\mathrm{R}_{\mathrm{e}}$ is the only dimensionless parameter in the equation. The assumption of $\operatorname{Re}<<1$ is very interesting and relevant to many practical problems.

At high Reynolds number, the kinetic energy dissipation rate $\mathcal{E}$ is equal to the fluctuating vorticity multiplied by the viscosity. The turbulent kinetic energy equation for K-Epsilon model is

$\frac{\partial k}{\partial t}+\operatorname{div}(\rho \underline{u} k)=\operatorname{div}\left(\left[\mu_{\text {lam }}+\frac{\rho \nu_{t}}{\sigma_{k}}\right] \operatorname{grad} k\right)+\rho \nu_{t} G-\rho \epsilon$

The turbulent generation rate is represented by $\mathrm{G}$

$$
\begin{aligned}
& G=2\left(\left[\frac{\partial u}{\partial x}\right]^{2}+\left[\frac{\partial v}{\partial y}\right]^{2}+\left[\frac{\partial w}{\partial z}\right]^{2}\right)+\left(\frac{\partial u}{\partial y}+\frac{\partial v}{\partial x}\right)^{2}+ \\
& \left(\frac{\partial u}{\partial z}+\frac{\partial w}{\partial x}\right)^{2}+\left(\frac{\partial w}{\partial y}+\frac{\partial v}{\partial z}\right)^{2}
\end{aligned}
$$

The Kolmogorov-Prandtl expression used for the turbulent viscosity in the implementation of this model is

$$
v_{t}=C_{\mu} \frac{k^{2}}{\epsilon}
$$

\section{Results}

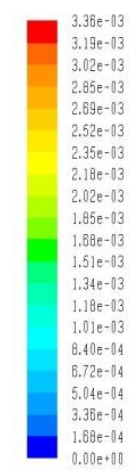

Pathlines Colored by Velocity Magnitude $(\mathrm{m} / \mathrm{s}$

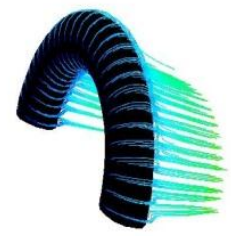

Fig 2. Path lines of Velocity magnitude $(\mathrm{Re}=3000)$

Figure 2 shows the velocity magnitude path lines for Reynolds number 3000 . It is very clear that there is no formation of wakes near the ring.
Figure 5 shows the path lines of velocity magnitude for Reynolds number 100000 . The formation of wakes can be seen near the ring.

Figures 3 and 6 shows the path lines of turbulent kinetic energy at Reynolds numbers 3000 and 100000 respectively.

The formation of wakes is seen near the ring and also mixing is so stronger such that wakes recover so quickly for Reynolds number 100000 than for Reynolds number 3000. This is very clear from Figures 4 and 7.
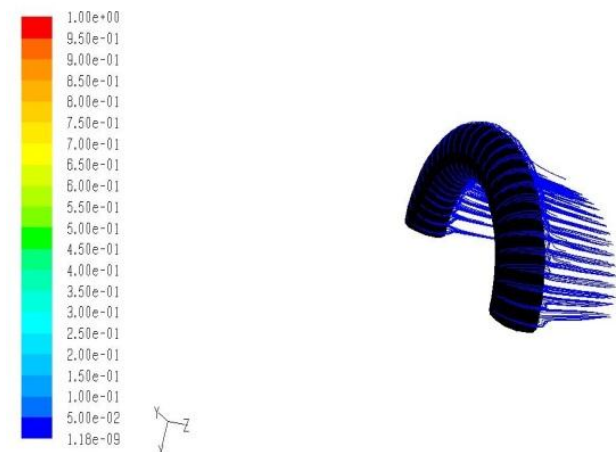

Pathlines Colored by Turbulent Kinetic Energy $(k)(\mathrm{m} 2 / \mathrm{s} 2$ FLUENT 6.3 (3),

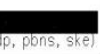

Fig 3. Path lines of Turbulent kinetic Energy $(\mathrm{Re}=3000)$
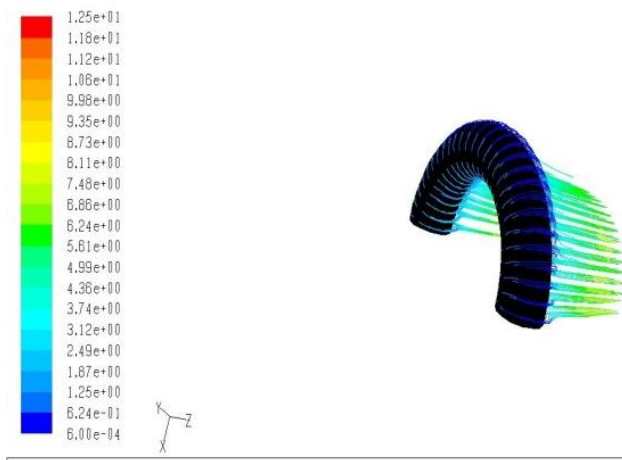

Pathlines Colored by Turbulent Viscosity $(\mathrm{kg} / \mathrm{m}-\mathrm{s})$ FLUENT 6.3 (3⿻

Fig 4. Path lines of Turbulent Viscosity $(\mathrm{Re}=3000)$
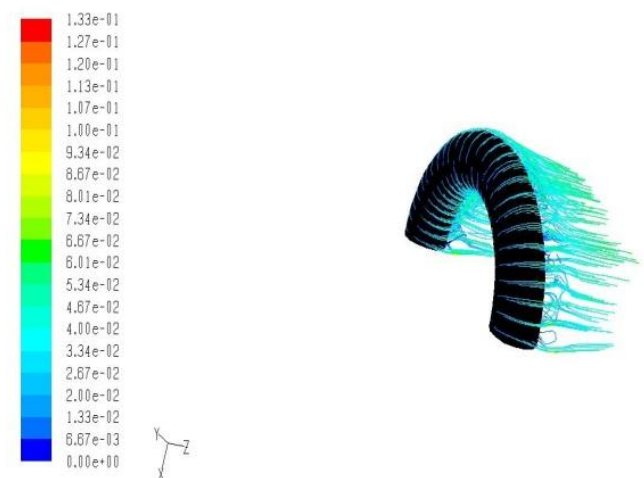

Pathlines Colored by Velocity Magnitude $(\mathrm{m} / \mathrm{s})$ FLUENT 6.3130

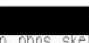

Fig 5. Path lines of Velocity Magnitude $(\mathrm{Re}=100000)$ 


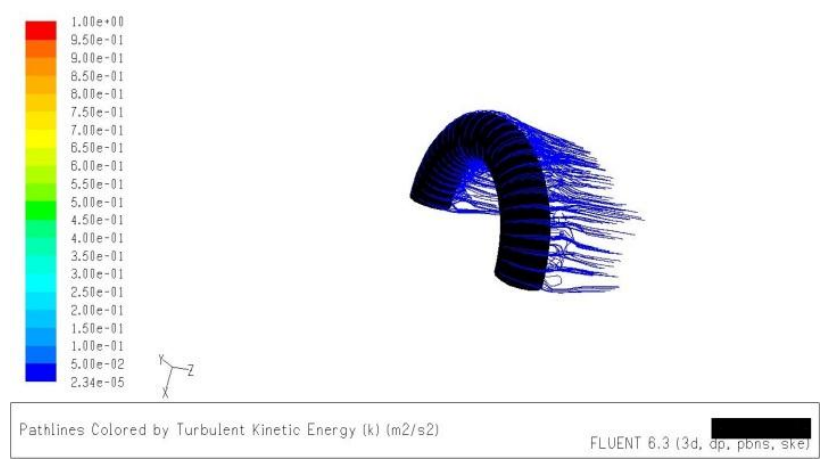

Fig 6. Path lines of Turbulent kinetic Energy $(\mathrm{Re}=100000)$

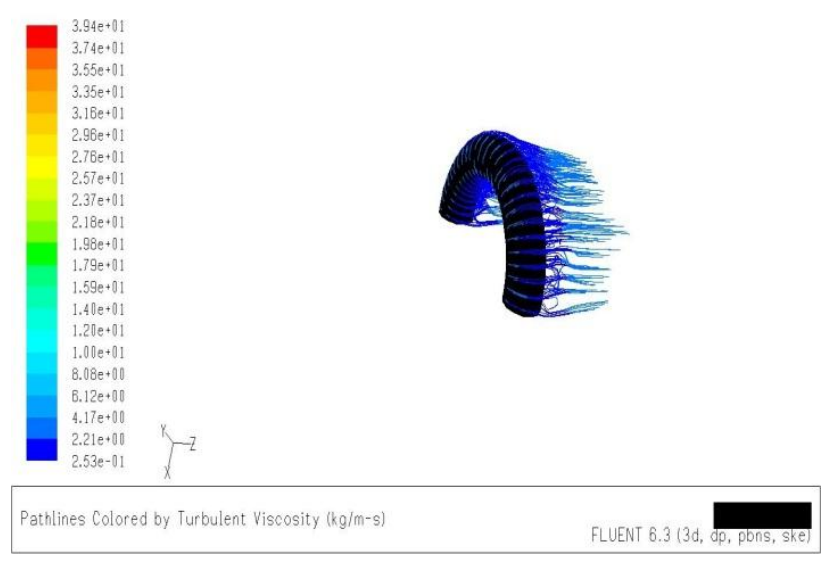

Fig 7. Path lines of Turbulent Viscosity $(\mathrm{Re}=100000)$

\section{Conclusion}

The flow characteristics are quite different between two different Reynolds numbers of $R_{e}=3000$ and $10^{5}$. At $R_{e}=$ 3000 , elongation of shear layer is observed to form a cylindrical vortex sheet in streamwise direction. At $\mathrm{R}_{\mathrm{e}}=$
$10^{5}$, the instability of shear layer occurs right behind the ring and near wake, the flow becomes turbulent. The flow behind the ring at $R_{e}=3000$ is nearly laminar with few vertices. Hence at $R_{e}=3000$, the base pressure is high than at $R_{e}=10^{5}$. At $R_{e}=10^{5}$, mixing is much stronger near wake than at $\mathrm{R}_{\mathrm{e}}=3000$ and thus more quickly the wake recovers.

\section{References}

Beaudan.P, Moin.P(1994), Numerical experiments on the flow past a circular cylinder at sub-critical Reynolds number Report No. TF-62, Department of Mechanical Engineering, Stanford University.

Constantinescu.G.S, Squires.K.D(2000), LES and DES investigations of turbulent flow over a sphere. AIAA paper 2000-0540.

Jeong.J, Hussain.F(1995), On the identification of a vortex. J. Fluid Mech. 285, 69-94.

Johnson.T.A, Patel.V.C(1999), Flow past a sphere up to a Reynolds number of 300. J. Fluid Mech. 378, 19-70.

Kim.h.j, durbin. P.A(1988), Observations of the frequencies in a sphere wake and of drag increase by acoustic excitation. Phys. Fluids 31, 3260-3265.

Kim.J, Kim.D, Choi.H(2001), An immersed-boundary finite volume method for simulations of flow in complex geometries. J. Comput. Phys. 171, 132-150.

Mittal.R(1999), Planar symmetry in the unsteady wake of a sphere. AIAA J. 37, 388-390.

Sakamoto.H, Haniu.H(1990), A study on vortex shedding from spheres in a uniform flow. J. Fluid Eng. 112, 386-392.

Tolstykh.A.I(1994), High accuracy non-centered compact difference schemes for fluid dynamics applications. World Scientific, Singapore.

Tomboulides.A(1993), Direct and large-eddy simulation of wake flows: flow past a sphere. Ph.D. dissertation, Princeton University

Zhong.X(1998), High-order finite-difference schemes for numerical simulation of hypersonic boundary-layer transition. J. Comput. Phys. 144, 662-709. 